\title{
Anharmonic softening of Raman active phonons in Iron-Pnictides; estimating the Fe isotope effect due to anharmonic expansion.
}

\author{
M. Granath* \\ Department of Physics, University of Gothenburg, Göteborg 41296 Sweden \\ J. Bielecki, J. Holmlund, and L. Börjesson \\ Department of Applied Physics, Chalmers University of Technology, Göteborg 41296 Sweden
}

(Dated: September 14, 2021)

\begin{abstract}
We present Raman measurements on the iron-pnictide superconductors $\mathrm{CeFeAsO}_{1-x} \mathrm{~F}_{x}$ and $\mathrm{NdFeAsO}_{1-x} \mathrm{~F}_{x}$. Modeling the Fe-As plane in terms of harmonic and a cubic anharmonic Fe-As interaction we calculate the temperature dependence of the energy and lifetime of the Raman active Fe $B_{1 g}$ mode and fit to the observed energy shift. The shifts and lifetimes are in good agreement with those measured also in other Raman studies which demonstrate that the phonon spectrum, at least at small wave numbers, is well represented by phonon-phonon interactions without any significant electronic contribution. Even at zero temperature there is a non negligent effect of interactions on the phonon energy, which for the Fe $B_{1 g}$ mode corresponds to $6 \mathrm{~cm}^{-1}$, or $3 \%$ of the total energy of the mode. We also estimate the anharmonic expansion from Fe $(56 \rightarrow 54)$ isotope substitution to $\Delta a \approx 5.1 \cdot 10^{-4} \AA$ and $\Delta d_{F e-A s} \approx 2.5 \cdot 10^{-4} \stackrel{\AA}{ }$ and the shift of harmonic zero point fluctuations of bond lengths $\left\langle\Delta x^{2}>\lesssim 3 \cdot 10^{-5} \AA^{2}\right.$, giving a total relative average decrease of electronic hopping integrals of $|\delta t| / t \lesssim 2.0 \cdot 10^{-4}$. For a non-phonon mediated weak coupling superconductor this gives an isotope exponent $\alpha \sim 10^{-2}$. The results poses a serious challenge for any theory of superconductivity in the pnictides that does not include electron-phonon interactions to produce a sizable Fe-isotope effect.
\end{abstract}

\section{INTRODUCTION}

The recently discovered iron-pnictide high temperature superconductors ${ }^{11}$ have several features in common with the much studied cuprate superconductors. In addition to their overall quasi-two dimensional nature such features include commensurate antiferromagnetism in close proximity to or even coexisting with superconductivity. Intriguingly, there is also a common tendency for breaking of the fourfold rotational symmetry of the crysta ${ }^{[2}$ which in the case of the cuprates is most likely an intrinsic electronic property related to the strong correlations. ${ }^{3}$ For the pnictides (as well as in some cases for the cuprates) there is at low doping a crystal symmetry breaking from tetragonal to orthorombic which is closely connected to an accompanying spin density wave (SDW) order. ${ }^{45}$ This interplay between atomic and electronic degrees of freedom naturally leads to the question: What is the role of phonons for the electronic properties of these systems? Focusing on the pnictides, results from density functional theory (DFT) ${ }^{6 / 7}$ are credible as they give an electronic spectral distribution consistent with photoemission experiment $\sqrt{8}$ but have also found that the electron-phonon coupling is much too weak to explain the high $\mathrm{T}_{c}{ }^{[7}$ Challenging these findings was the recent report of an isotope shift of $\mathrm{T}_{c}$ (and $\mathrm{T}_{S D W}$ ) when substituting ${ }^{56} \mathrm{Fe}$ with ${ }^{54} \mathrm{Fe}$ with an exponent of $\alpha=-\frac{d \ln T_{c}}{d \ln m}=0.4$ close to the BCS value of 0.5 for a pure iron mode ${ }^{9}$ The mechanism for superconductivity in the pnictides is a major unresolved issue and it is clearly essential to understand better the lattice-electron interplay.

In this paper we measure and model one particular Ra- man active phonon which lives primarily on Fe atoms and thus common to all iron-pnictide superconductors. The purpose is to study the anharmonic structure of the FeAs plane with two main objectives: First, to investigate whether any non-phonon contributions are necessary to describe the temperature dependence of the phonon energy and lifetime. Second, to estimate the magnitude of the lattice expansion that follows from substitution with a lighter mass and the corresponding changes in electronic hopping integrals, thus investigating the plausibility of a non-phonon related isotope effect in the pnictides $\frac{10 \mid 11}{10}$

In brief, we model the harmonic spectrum of the As-Fe plane and use standard Greens function methods to calculate the self energy of the $\mathrm{Fe} \mathrm{B}_{1 g}$ Raman phonon to second order in the cubic anharmonic FeAs interatomic coupling. The harmonic spectrum is based on a minimal parameter fit to spectra derived within the local density approximation (LDA) of DFT but the calculation of the temperature dependent contribution from phonon-phonon interactions is beyond the capabilities of that method. We present Raman results on (Nd,Ce)FeAsO ${ }_{1-x} \mathrm{~F}_{x}$ and estimate the anharmonic coupling strength by fitting our model calculations to the measured temperature dependent energy shift. Also the width agrees well with Raman measurements on $\mathrm{CaFe}_{2} \mathrm{As}_{2} \frac{\sqrt{12}}{2}$ thus effectively ruling out any significant electronic contribution to the broadening. Based on the magnitude of the anharmonic coupling we estimate the isotope $\left({ }^{56} \mathrm{Fe} \rightarrow{ }^{54} \mathrm{Fe}\right)$ shift of the lattice parameters to $\lesssim 2 \cdot 10^{-4}$ and calculate a similar relative decrease of interatomic hopping integrals. Harmonic zero point fluctuations give an even smaller isotope shift of hopping 


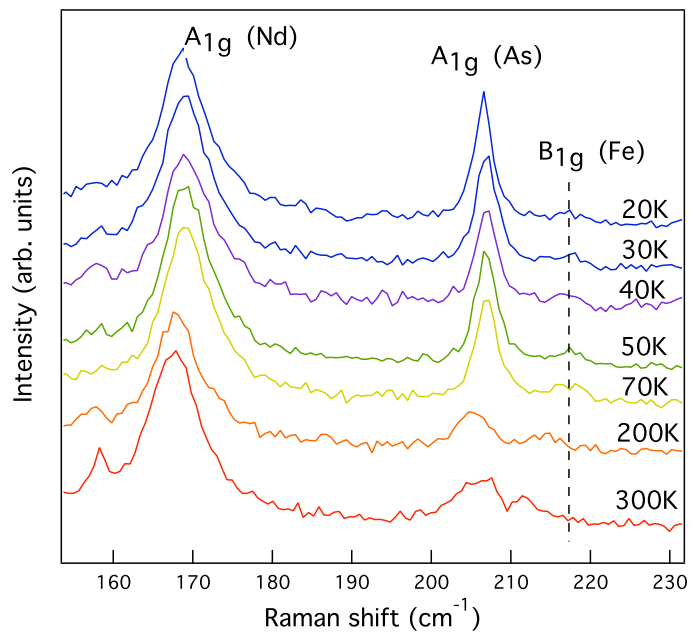

FIG. 1: (Color online) Temperature dependent Raman spectra for $\mathrm{NdFeAsO}_{1-x} \mathrm{~F}_{x}(x=.12)$ showing three Raman active phonon modes labeled by their symmetry and main atomic displacement.

integrals. In weak coupling theory correspondingly small changes in the electronic density of states (DOS) is expected to give an isotope exponent $\alpha \sim 10^{-2}$.

\section{RAMAN SPECTRA}

Raman spectra between $100 \mathrm{~cm}^{-1}$ and $400 \mathrm{~cm}^{-1}$ were collected at temperatures ranging from $20 \mathrm{~K}$ to $300 \mathrm{~K}$ for polycrystalline samples of $\mathrm{CeFeAsO}_{1-x} \mathrm{~F}_{x}(x=0.16)$ and $\mathrm{NdFeAsO}_{1-x} \mathrm{~F}_{x}(x=0.12){ }^{14}$ For sample preparation and characterization see Chen at al ${ }^{[15}$ All spectra were recorded using a Dilor-XY800 spectrometer in dou-

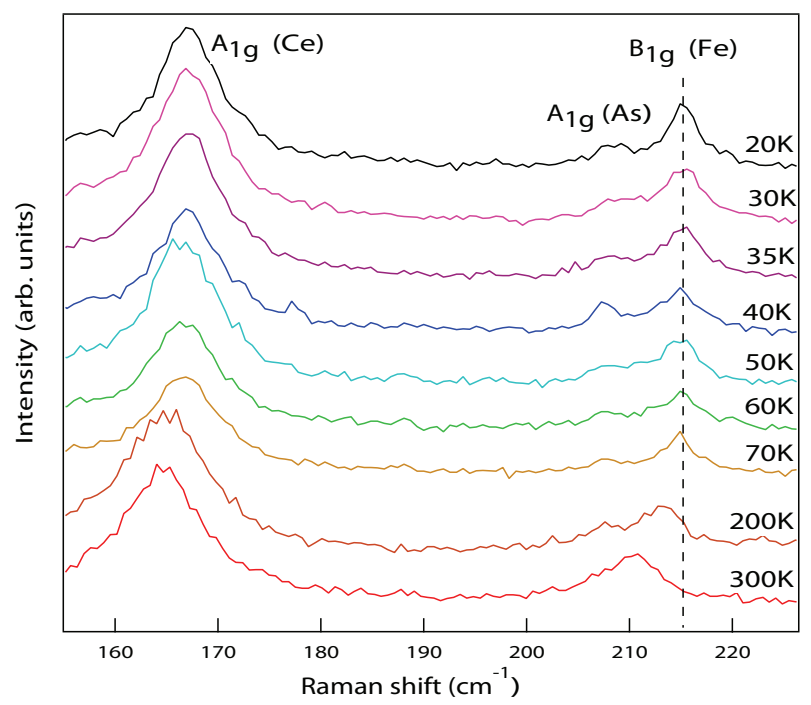

FIG. 2: (Color online) Raman spectra for $\mathrm{CeFeAsO}_{1-x} \mathrm{~F}_{x}$ $(x=.16)$. ble subtractive mode. In all scans the $514.5 \mathrm{~nm}$ line from a $\mathrm{Ar}+$ laser used with a power of less than $1 \mathrm{~mW}$ was focused onto the samples with a spot size less than $2 \mu \mathrm{m}$. The samples were installed in a LHe cooled cryostat. We observe three Raman active modes with energies around 170, 210 and $220 \mathrm{~cm}^{-1}$, see Fig. 1 and Fig. 2, in agreement with other Raman studies, $\frac{13}{13}$ that are identified as $(\mathrm{Ce} / \mathrm{Nd})-\mathrm{A}_{1 g}, \mathrm{As}_{\mathrm{s}} \mathrm{A}_{1 g}$, and $\mathrm{Fe}-\mathrm{B}_{1 g}$ modes respectively. Also in agreement with earlier studies we found no effect on the phonon energies from crossing into the superconducting phase $\left(\mathrm{T}_{c}=35 \mathrm{~K} / 45 \mathrm{~K}\right.$ for $\left.\mathrm{Ce} / \mathrm{Nd}\right)$. As discussed in the introduction we will be interested in analysing the temperature dependence of the energy as extracted in Fig. 3 and for modeling purposes we consider only the $\mathrm{Fe}-\mathrm{B}_{1 g}\left(x^{2}-y^{2}\right)$ mode which lives primarily in the Fe-As plane.
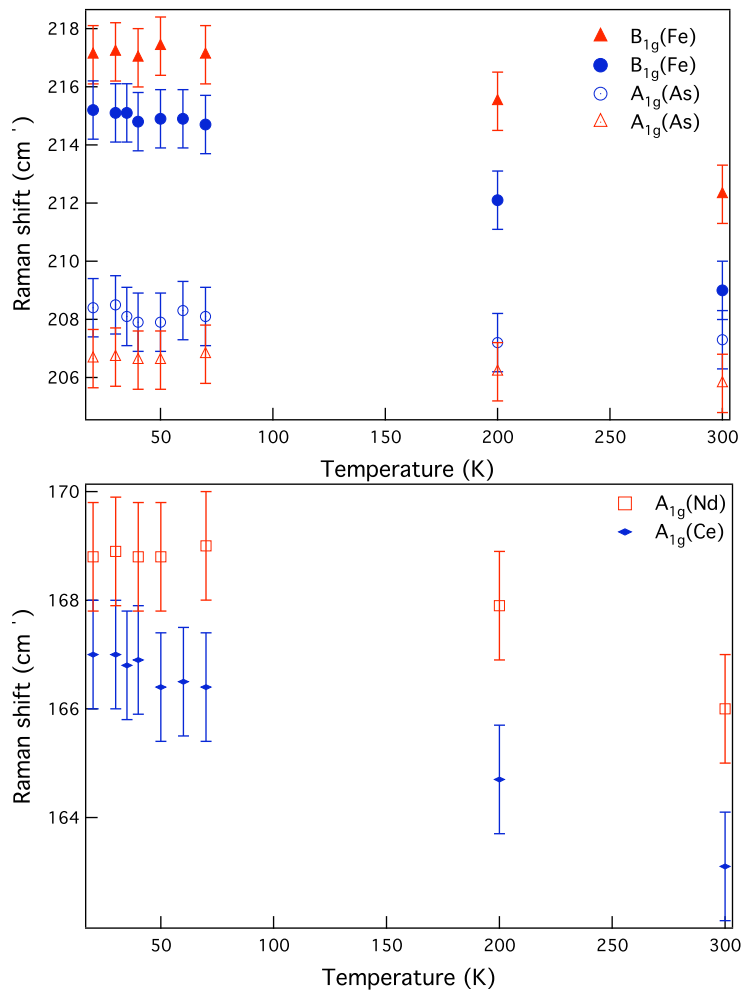

FIG. 3: (Color online) Temperature dependence of the phonon peak position for $\mathrm{NdFeAsO}_{0.88} \mathrm{~F}_{0.12}$ (red) as extracted from Fig. 1 and correspondingly for $\mathrm{CeFeAsO}_{0.84} \mathrm{~F}_{0.16}$ (blue) from Fig. 2 .

\section{MODELING}

The temperature dependence of the lifetime and energy of a phonon is ordinarily due to phonon-phonon interactions that arise from anharmonic interatomic potentials. In general the cubic anharmonicity is the dominant term 16 and we consider only this. The Raman $(\vec{q}=0)$ intensity for Stokes scattering for the mode $j$ at frequency 
$\omega$ is given by $I_{S}(j, \omega) \propto-(1+n(\omega)) \operatorname{Im} \mathcal{D}_{\text {ret }}(\vec{q}=0, j, \omega)^{17}$ where $\mathcal{D}_{\text {ret }}$ is the retarded phonon Greens function which to linear order in the self energy $\Pi=\Delta-i \Gamma$ (minus sign by convention) gives

$$
I_{S}(j, \omega) \propto \frac{(1+n(\omega)) \Gamma(\overrightarrow{0}, j, \omega)}{\left[\omega_{0}(\overrightarrow{0}, j)+\Delta(\overrightarrow{0}, j, \omega)-\omega\right]^{2}+\Gamma^{2}(\overrightarrow{0}, j, \omega)}
$$

where $n(\omega)=\left(e^{\hbar \omega / k_{B} T}-1\right)^{-1}$ is the Bose occupation factor. The measured width (FWHM) is thus ideally given by $\Gamma$ and the shift by $\Delta$. We calculate the self energy to second order in the interaction $H_{A}=$ $\frac{1}{6} \sum_{\left\{\vec{q}_{i}\right\},\left\{j_{i}\right\}} V\left(\vec{q}_{1}, j_{1} ; \vec{q}_{2}, j_{2} ; \vec{q}_{3}, j_{3}\right) A_{\vec{q}_{1}, j_{1}} A_{\vec{q}_{2}, j_{2}} A_{\vec{q}_{3}, j_{3}} \delta_{\sum \vec{q}_{i}, 0}$ where $A_{\vec{q}, j}=a_{\vec{q}, j}+a_{-\vec{q}, j}^{\dagger}$ is the phonon operator ${ }^{16} V$ are the matrix elements

$$
V\left(\vec{q}_{1}, j_{1} ; \vec{q}_{2}, j_{2} ; \vec{q}_{3}, j_{3}\right)=\sum_{r_{1}, r_{2}, r_{3}, \vec{R}_{1}, \vec{R}_{2}, \vec{R}_{3}}\left[\prod_{i=1}^{3}\left(\left(\frac{\hbar}{2 M_{r_{i}} \omega\left(\vec{q}_{i}, j_{i}\right)}\right)^{\frac{1}{2}} e^{i \vec{q}_{i} \cdot \vec{R}_{i}} \vec{e}_{\vec{q}_{i}, j_{i}}\left(r_{i}\right) \cdot \nabla\right)\right] \phi\left(r_{1}, r_{2}, r_{3}, \vec{R}_{1}, \vec{R}_{2}, \vec{R}_{3}\right)
$$

where the derivatives act on $\phi$, the interatomic potential, with $r_{i}$ the atomic positions within unit cell $\vec{R}_{i}$ and $\vec{e}_{\vec{q}_{i}, j_{i}}\left(r_{i}\right)$ are the displacement vectors of the respective mode. The expression thus amounts to the third derivate with respect to phonon displacements of the atomic positions. The iron atoms are only weakly coupled to the $(\mathrm{Ce}, \mathrm{Nd}) \mathrm{O}$ layer and since the phonon dispersions in the c-direction are weak ${ }^{7}$ we can model the the $\mathrm{Fe}_{1 g}$ mode by considering only the Fe-As plane. (This also makes the procedure quasi-universal as it only depends on the properties of this plane which is common to all iron-pnictide superconductors.) For the harmonic problem we use a minimal spring model (Fig. 4) with nearest neighbor Fe-As potential $\frac{k}{2} \delta r^{2}$, nearest neighbor FeFe potential $\frac{k^{\prime}}{2} \delta r^{\prime 2}$, and in-plane As-As coupling $\frac{k^{\prime \prime}}{2} \delta r^{\prime \prime 2}$ with $\delta r, \delta r^{\prime}$, and $\delta r^{\prime \prime}$ the deviations from the respective equilibrium distances. For the Fe-As coupling we also add a cubic term $-\frac{g}{6} \delta r^{3}$, where the magnitude of $g$ is to be estimated from the experimental fit. The Raman active $\mathrm{B}_{1 g}$ is here a pure Fe mode as depicted in Fig. 4 with energy $\omega_{B_{1 g}}=\sqrt{\frac{4 k \sin ^{2} \theta}{m}}(m=56 u)$ and to get $\omega_{B_{1 g}}=220 \mathrm{~cm}^{-1}$ we take $k=8.7 \mathrm{eV} / \AA^{2}$, using $\theta=35^{0 ! 5}$ Values $k^{\prime}=0.3 k$ and $k^{\prime \prime}=0.2 k$ gives a lower edge of the accoustic branches in good agrement with LDA calculations. 7 Using this phonon spectrum we calculate the interaction matrix elements through Eq. 2 and the cubic potential. Sampling the coupling $V\left(\overrightarrow{0}, \vec{F} e_{B 1 g} ; \vec{q}, j_{1} ;-\vec{q}, j_{2}\right) \equiv V_{0}\left(\vec{q}, j_{1}, j_{2}\right)$ over the Brillouin zone shows that except at high symmetry points all 12 modes are coupled quite isotropically and does not vary strongly with $\vec{q}$ if the frequency dependence is included, giving

$$
V_{0}\left(\vec{q}, j_{1}, j_{2}\right) \approx \kappa g\left(\frac{\hbar}{2 m \omega_{B_{1 g}}}\right)^{3 / 2} \equiv V_{0}
$$

with the numerical value $\kappa=0.10$.

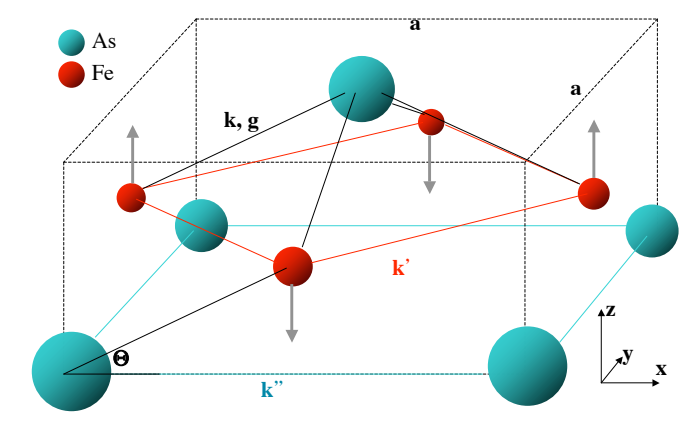

FIG. 4: (Color online) Schematic of the Fe-As plane with in-plane lattice parameter $a$. The harmonic interatomic couplings are $k, k^{\prime}$ and $k^{\prime \prime}$ and cubic coupling $g$ as described in the text. Arrows indicate the Fe $\mathrm{B}_{1 g}$ mode.

\section{A. Phonon self energy}

Now we are ready to address the frequency shift by calculating the self-energy to second order in $V_{0}$. The self energy is given by the bubble diagram ${ }^{16}$ with imaginary part 


$$
\begin{aligned}
& \Gamma(\omega)=\frac{\pi}{2 N \hbar^{2}} \sum_{\vec{q}, j_{1}, j_{2}}\left|V_{0}\left(\vec{q}, j_{1}, j_{2}\right)\right|^{2}\left[\left(1+n\left(\omega_{\vec{q}, j_{1}}\right)+n\left(\omega_{\vec{q}, j_{2}}\right)\right)\left[\delta\left(\omega-\omega_{\vec{q}, j_{1}}-\omega_{\vec{q}, j_{2}}\right)-\delta\left(\omega+\omega_{\vec{q}, j_{1}}+\omega_{\vec{q}, j_{2}}\right)\right]\right. \\
& \left.+\left(n\left(\omega_{\vec{q}, j_{1}}\right)-n\left(\omega_{\vec{q}, j_{2}}\right)\right)\left[\delta\left(\omega+\omega_{\vec{q}, j_{1}}-\omega_{\vec{q}, j_{2}}\right)-\delta\left(\omega-\omega_{\vec{q}, j_{1}}+\omega_{\vec{q}, j_{2}}\right)\right]\right]
\end{aligned}
$$

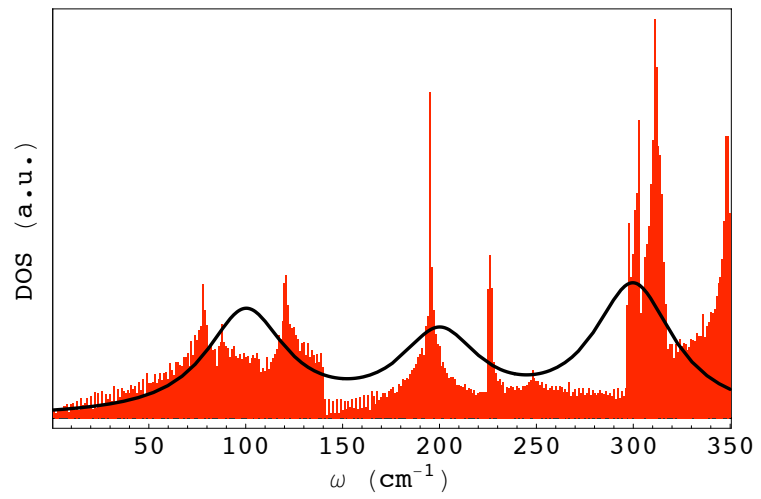

FIG. 5: (Color online) Phonon density of states of the isolated Fe-As plane calculated within the harmonic model discussed in the text and its approximation in terms of three Lorentzians (solid curve) that contain 4,3,5 modes with increasing energy respectively.

(The phonon-difference process, the second term, vanishes at zero temperature but can give a significant contribution at finite temperature.) The simplest standard way to evaluate this expression is to assume that the scattering is diagonal in the modes $V_{0} \sim \delta_{j_{1}, j_{2}}$, the Klemens model, $\frac{18}{18}$ which gives a characteristic temperature dependence $\Gamma(\omega) \sim(1+2 n(\omega / 2))$. As discussed previously we find instead that the scattering is approximately isotropic between modes and we need to do a more careful analysis. In principle we could calculate this expression numerically at each temperature using our numerical phonon spectrum but instead we will use an approximate Lorentzian fit for the phonon DOS with the advantage of giving an analytic expression for both $\Gamma$ and $\Delta$ including the full temperature dependence. The calculated phonon DOS, plotted in Fig. 5, consists of three regions of high density centered around $w_{1} \approx 100 \mathrm{~cm}^{-1}, w_{2} \approx 200 \mathrm{~cm}^{-1}$, $w_{3} \approx 300 \mathrm{~cm}^{-1}$. The lower part contains $m_{1}=4$ modes, the intermediate part contains $m_{2}=3$ modes and the upper part $m_{3}=5$ modes giving

$$
\rho(\omega) \approx \frac{N}{\pi} \sum_{i=1}^{3} m_{i} \frac{\gamma}{\left(w-w_{i}\right)^{2}+\gamma^{2}} \equiv \sum_{i} \rho_{i}(\omega)
$$

which is thus normalized by $\int_{-\infty}^{\infty} \rho(\omega)=12 N$ and where we estimate $\gamma=25 \mathrm{~cm}^{-1}$. To proceed we evaluate the occupation factor $n(\omega)$ at the peak position of the respective DOS and assume a q-independent spectral weight giving $\Gamma$ as an integral over all pairs of peaks of the spectral weight with the result

$$
\begin{aligned}
\Gamma_{0}(\omega)=\frac{1}{2 \hbar^{2}} V_{0}^{2} \sum_{i, j=1}^{3} m_{i} m_{j} \quad & {\left[\left(1+n\left(\omega_{i}\right)+n\left(\omega_{j}\right)\right)\left[\frac{2 \gamma}{\left(\omega-\omega_{i}-\omega_{j}\right)^{2}+4 \gamma^{2}}-\frac{2 \gamma}{\left(\omega+\omega_{i}+\omega_{j}\right)^{2}+4 \gamma^{2}}\right]\right.} \\
& \left.+\left(n\left(\omega_{i}\right)-n\left(\omega_{j}\right)\right)\left[\frac{2 \gamma}{\left(\omega+\omega_{i}-\omega_{j}\right)^{2}+4 \gamma^{2}}-\frac{2 \gamma}{\left(\omega-\omega_{i}+\omega_{j}\right)^{2}+4 \gamma^{2}}\right]\right]
\end{aligned}
$$

The expression is naturally understood as the scattering of the mode at $\omega$ into two modes within the same or different DOS peaks (or the corresponding difference process $)$. Note that $\Gamma_{0}\left(\omega_{B_{1 g}}\right)$ will be dominated by scattering into the low-energy peak $(i=j=1)$ which will give a temperature dependence close to the Klemens model $\Gamma(\omega) \sim(1+2 n(\omega / 2)) ! \frac{18}{}$ Using the Kramers-Kronig relation $\Delta(\omega)=-\frac{1}{\pi} \int_{-\infty}^{\infty} \frac{\Gamma\left(\omega^{\prime}\right)}{\omega^{\prime}-\omega} d \omega^{\prime}$, gives

$$
\begin{aligned}
\Delta_{0}(\omega)=\frac{1}{2 \hbar^{2}} V_{0}^{2} \sum_{i, j=1}^{3} m_{i} m_{j} \quad & {\left[\left(1+n\left(\omega_{i}\right)+n\left(\omega_{j}\right)\right)\left[\frac{\left(\omega-\omega_{i}-\omega_{j}\right)}{\left(\omega-\omega_{i}-\omega_{j}\right)^{2}+4 \gamma^{2}}-\frac{\left(\omega+\omega_{i}+\omega_{j}\right)}{\left(\omega+\omega_{i}+\omega_{j}\right)^{2}+4 \gamma^{2}}\right]\right.} \\
& \left.+\left(n\left(\omega_{i}\right)-n\left(\omega_{j}\right)\right)\left[\frac{\left(\omega+\omega_{i}-\omega_{j}\right)}{\left(\omega+\omega_{i}-\omega_{j}\right)^{2}+4 \gamma^{2}}-\frac{\left(\omega-\omega_{i}+\omega_{j}\right)}{\left(\omega-\omega_{i}+\omega_{j}\right)^{2}+4 \gamma^{2}}\right]\right]
\end{aligned}
$$




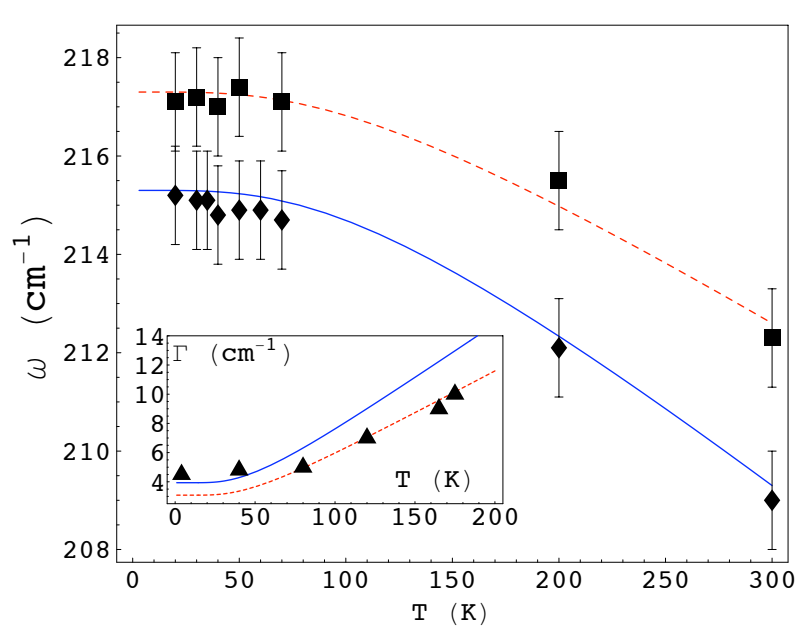

FIG. 6: (Color online) Fit of the model frequency shift $\left(\Delta_{0}\right)$ to data for $\mathrm{NdFeAsO}{ }_{88} \mathrm{~F}_{.12}$ (boxes) and $\mathrm{CeFeAsO}{ }_{84} \mathrm{~F}_{.16}$ (diamonds). Inset are the corresponding model lifetimes $\left(\Gamma_{0}\right)$ together with linewidth data (triangles) on $\mathrm{CaFe}_{2} \mathrm{As}_{2}$ from Choi et al. 12 .

\section{DISCUSSION}

Figure 6 shows the fit from equation 7 to the experimental values using $\omega=\omega_{0}+\Delta_{0}\left(\omega_{B_{1 g}}\right)$, with fitting parameters $\omega_{0}$ and $g\left(\Delta_{0} \sim g^{2}\right)$. For the Nd sample we find $\omega_{0}=221.7 \mathrm{~cm}^{-1}$ and $\mathrm{g}=103 \mathrm{eV} / \AA^{3}$ and for the Ce sample $\omega_{0}=221.0 \mathrm{~cm}^{-1}$ and $g=116 \mathrm{eV} / \AA^{3}$, where $\omega_{0}$ is close to the assumed harmonic value $\omega_{B_{1 g}}=220 \mathrm{~cm}^{-1} \underline{19}$ It is interesting to note that we find $\Delta_{0}(T=0) \approx 6 \mathrm{~cm}^{-1}$, i.e. even at zero temperature phonon-phonon interactions give a finite (here $3 \%$ ) contribution to the phonon energy, an effect which LDA calculations of phonon energies based on linear response neglects.

Due to the polycrystalline nature of our samples we are not able to find reliable phonon widths and compare instead our theoretical results to Raman studies by Choi et al ${ }^{[12}$ on $\mathrm{CaFe}_{2} \mathrm{As}_{2}$. In that material the $\mathrm{B}_{1 g}$ mode has lower energy but the temperature dependence is similar and the width correspond well with our calculations without any additional fit. The temperature dependence is in fact just the Klemens model but the strength of the calculation is that only one parameter $g$ gives both the shift and the width. In Re ${ }^{12}$ the linewidth variations were tentatively assigned to changes in the electronic scattering below $\mathrm{T}_{S D W}$. These speculations we can quite definitely rule out; the anharmonic contribution accounts to good accuracy for both the shift and linewidth variations of this mode.21

\section{A. Lattice expansion}

The cubic coupling will give rise to an expansion of the lattice that we estimate by considering an isolated
Fe-As bond for which $<0|\delta r| 0>=\frac{\hbar g}{4(m *)^{1 / 2} k^{3 / 2}}$ with $m^{*}=M m /(m+M)$. We include also a nearest neighbor Fe-Fe anharmonic coupling $g^{\prime}$ with the same relative strength $\left(g^{\prime}=g\left(k^{\prime} / k\right)^{3 / 2}\right)$. With this the isotope substitution ${ }^{56} \mathrm{Fe} \rightarrow{ }^{54} \mathrm{Fe}$ gives an expansion of the in-plane lattice parameter $\Delta a=5.1 \cdot 10^{-4} \AA$ and Fe-As distance $\Delta d_{F e-A s}=2.5 \cdot 10^{-4} \AA$, which compared to $a \approx 4 \AA$ and $d_{F e-A s} \approx 2.5 \AA .5$ gives a relative expansion $\lesssim 2 \cdot 10^{-4}$.

What is the possible effect of a small lattice expansion on $T_{c}$ and $T_{S D W}$ ? There is a direct effect on the electronic hopping integrals $t$, and theories of an isotope effect based on this has been suggested for the cuprates and $\mathrm{C}_{60}$ as well the pnictides $\frac{1011124}{\text { Assuming }}$ $t=t_{0} e^{-q\left(r / r_{0}-1\right)}$ with $q \sim 1^{\sqrt{22}}$ and where $\vec{r}=r_{0} \hat{r}+\vec{\delta}$ with small displacement $\vec{\delta}$ we find $\frac{\delta t}{t_{0}}=-q \frac{\delta_{r}}{r_{0}}-\frac{q}{2} \frac{\delta_{\perp}^{2}}{r_{0}^{2}}+\frac{q^{2}}{2} \frac{\delta_{r}^{2}}{r_{0}^{2}}$ where $\delta_{r}=\vec{\delta} \cdot \hat{r}$ and $\delta_{\perp}=\vec{\delta} \times \hat{r}$. The linear term has only an anharmonic contribution whereas the quadratic terms will get contributions from zero point fluctuations in the harmonic approximation. We estimate $\delta^{2}$ from the ground state energy per atom $\epsilon_{0}=38.9 \mathrm{meV}$ and $\epsilon_{0}=39.2 \mathrm{meV}$ for ${ }^{56} \mathrm{Fe}$ and ${ }^{54} \mathrm{Fe}$ respectively and energy $\epsilon_{0} / 2$ per Fe-As bond. The fluctuation along a bond is given by $\frac{k}{2} \delta r^{2} \approx \epsilon_{0} / 4\left(k=8.7 e V / \AA^{2}\right)$, giving the difference $\delta_{r}^{2} \approx \Delta \epsilon_{0} /(2 k) \approx 2.3 \cdot 10^{-5} \AA^{2}$ and for the transverse fluctuations $\delta_{\perp}^{2} \approx 2 \delta_{r}^{2} \approx 4.6 \cdot 10^{-5} \AA^{2} \stackrel{25}{25}$ The contribution to the shift of the hopping integrals is partially canceled by the different signs and in total smaller by an order of magnitude compared to the anharmonic contribution.

\section{B. Isotope effect}

Consider a weak coupling (non phonon mediated) SC or SDW transition with $T_{c} \sim \Omega e^{-1 / N(0) V}$, where $\Omega$ is the relevant energy cut-off, which may be the magnon energy for spin fluctuation mediated pairing, $V$ is the effective interaction and $N(0)$ the relevant electronic DOS at the Fermi energy. Focusing on the contribution from the DOS, $N(0) \sim 1 / t$ gives the isotope exponent $\alpha=-\frac{d \ln T_{c}}{d \ln m} \approx-\frac{1}{N(0) V} \frac{d \ln N(0)}{d \ln m} \approx \frac{0.5 \cdot 10^{-2}}{N(0) V} \approx 10^{-2}$. (Assuming, $N(0) V \approx 0.5$. It cannot be much smaller to get a high $\mathrm{T}_{c}$.) The interaction strength and $\Omega$ may also depend on the hopping integrals and give isotope shifts of similar magnitude. Although this is a simple analysis we expect the order of magnitude estimate to be relevant to any purely electronic microscopic model containing inter and intra orbital hopping integrals and interactions that only depend indirectly on the lattice parameters.

Alternatively, we may relate the change of lattice parameter to a corresponding pressure of $d P=\frac{3 \Delta a}{a} / \beta \approx$ $0.4 \mathrm{kbar}$ through the compressibility $\beta=-\frac{d \ln V}{d P} \approx 1.0$. $10^{-3} / \mathrm{kbar}^{26}$ (We have no estimate of the c-axis change related to the in-plane expansion but only assume that this is of similar relative magnitude.) Pressure dependence of $\mathrm{T}_{c}$ of around $0.2 \mathrm{~K} / \mathrm{kbar}$ has been reported in several materials at different dopings although close to 
optimal doping it appears that the effect is generally significantly smaller. ${ }^{27}$ Nevertheless, from these considerations we find an upper estimate of the isotope exponent $\alpha \approx 0.06$.

Clearly something more sophisticated is needed to produce $\alpha=0.4$ as found experimentally in Ref 9 . Encouraging perhaps, the sign from the naive weak coupling analysis based on a change in the electronic DOS does agree with experiments and would naturally imply a similar exponent for both SC and SDW! ${ }^{28}$

\section{SUMMARY}

In summary, we find that the temperature dependent shift and width of a Raman active phonon is well represented by the anharmonic contribution, consistent with weak electron-phonon interactions. At the same time, we estimate the change of electronic hopping integrals due to Fe isotope substitution and find that these are too small to generate an isotope effect on $\mathrm{T}_{c}$ or $\mathrm{T}_{S D W}$ of the mag- nitude reported in ${ }^{9}$ without an explicit phonon contribution. These apparently contradictory results present a significant challenge for any theory of superconductivity in the iron-pnictides.

\section{ACKNOWLEDGEMENT}

We thank Professor Nan Lin Wang for providing the samples used in the Raman measurements.

Note added After the submission of this work x-ray diffraction (XRD) data on the samples used for the isotope experiments appeared (Ref. 9, supplemental). Changes in the lattice parameter of isotope substituted samples are the same within the experimental error of $\sim$ $1 \cdot 10^{-3} \stackrel{\circ}{A}$, which is greater than the change $\Delta a \approx 5 \cdot 10^{-4} \stackrel{\circ}{A}$ calculated here. There also appeared a report of a negative Fe-isotope effect with $\alpha=-0.18 .29$ Again, XRD data find the same in-plane lattice parameter within experimental error of $\sim 1 \cdot 10^{-3} \stackrel{\circ}{30}$
* Electronic address: mats.granath@physics.gu.se

1 Y. Kamihara, T. Watanabe, M. Hirano, and H. Hosono, J. Am. Chem. Soc. 130, 3296 (2008).

2 J. Zhao, Q. Huang, C. de la Cruz, Shiliang Li, J. W. Lynn, Y. Chen, M. A. Green, G. F. Chen, G. Li, Z. Li, J. L. Luo, N. L. Wang and Pengcheng Dai, Nature mat. 7, 953 (2008).

3 S.A.Kivelson, E. Fradkin, and V. Emery, Nature 393, 550 (1998).

4 J. Dong, H. J. Zhang, G. Xu, Z. Li, G. Li, W. Z. Hu, D. Wu, G. F. Chen, X. Dai, J. L. Luo, Z. Fang and N. L. Wang, Europhys. Lett. 83, 27006 (2008).

5 C. de la Cruz, Q. Huang, J. W. Lynn, Jiying Li, W. Ratcliff II, J. L. Zarestky, H. A. Mook, G. F. Chen, J. L. Luo, N. L. Wang and P. Dai, Nature 453, 899 (2008).

6 D.J. Singh and M.-H. Du, Phys. Rev. Lett. 100, 237003 (2008).

7 L. Boeri,O.V. Dolgov, and A. A. Golubov, Phys. Rev. Lett. 101, 026403 (2008).

8 Chang Liu, G. D. Samolyuk, Y. Lee, Ni Ni, Takeshi Kondo, A. F. Santander-Syro, S. L. Bud'ko, J. L. McChesney, E. Rotenberg, T. Valla, A. V. Fedorov, P. C. Canfield, B. N. Harmon, and A. Kaminski, Phys. Rev. Lett. 101, 177005 (2008); L. X. Yang, Y. Zhang, H. W. Ou, J. F. Zhao, D. W. Shen, B. Zhou, J. Wei, F. Chen, M. Xu, C. He, Y. Chen, Z. D. Wang, X. F. Wang, T. Wu, G. Wu, X. H. Chen, M. Arita, K. Shimada, M. Taniguchi, Z. Y. Lu, T. Xiang, D. L. Feng, Phys. Rev. Lett. 102, 107002 (2009).

9 R. H. Liu, T. Wu, G. Wu, H. Chen, X. F. Wang, Y. L. Xie, J. J. Yin, Y. J. Yan, Q. J. Li, B. C. Shi, W. S. Chu, Z. Y. Wu, X. H. Chen, Nature 459, 64 (2009).

10 D. S. Fisher, A. J. Millis, B. Shraiman, and R. N. Bhatt, Phys. Rev. Lett. 61, 482 (1988).

11 S. Chakravarty, S. A. Kivelson, M. I. Salkola, and S. Tewari, Science 256, 1306 (1992).

12 K.-Y. Choi D. Wulferding, P. Lemmens, N. Ni, S. L.
Bud'ko, and P. C. Canfield, Phys. Rev. B 78, 212503 (2008).

13 V.G. Hadjiev, M. N. Iliev, K. Sasmal, Y.-Y. Sun, and C. W. Chu, Phys. Rev. B 77 220505(R) (2008); A.P. Litvinchuk, V. G. Hadjiev, M. N. Iliev, Bing Lv, A. M. Guloy, and C. W. Chu, ibid 78 060503(R) (2008); Y. Gallais, A. Sacuto, M. Cazayous, P. Cheng, L. Fang, and H. H. Wen, ibid 78 132509 (2008).

14 We performed measurements also on undoped $(x=0)$ crystals of the same materials. Apart from small changes in phonon energies we found no significant difference in the Raman spectra between doped and undoped samples.

15 Chen Gen-Fu, Li Zheng, Wu Dan, Dong Jing, Li Gang, Hu Wan-Zheng, Zheng Ping, Luo Jian-Lin and Wang Nan-Lin, Chinese Phys. Lett. 25, 2235 (2008).

16 J. Menendez and M. Cardona, Phys. Rev. B 29, 2051 (1984); M. Calandra, M. Lazzeri, F. Mauri, Physica C, 456, 38 (2007).

17 W. Hayes and R. Loudon, Scattering of Light by Crystals (John Wiley and Sons, 1978).

18 P.G. Klemens, Phys. Rev. 148, 845 (1966).

19 Note that $\Delta_{0}(\omega)$ is a quite rapidly varying function of $\omega$ and sensitive to the input parameters $\omega_{i}$ and $\gamma$. Even avoiding any fine tuning of parameters we find that $g$ can vary by up to $50 \%$ from the values given above for moderate changes of our model DOS. However, values of $g \sim 100 \mathrm{ev} / \AA^{3}$ are also in line with estimates for $\mathrm{GaAs}^{20}$ which has a similar local lattice structure.

20 See, U. Pietsch and K. Paschke, Acta Cryst. B49, 822 (1993) and references therein.

21 In ${ }^{12}$ emphasis was put on changes at the SDW transition. While our results match that data for the low temperature orthorombic phase we have no explanation for the behavior above $\mathrm{T}_{S D W}$ where the $\mathrm{B}_{1 g}$ linewidth saturates and the As $\mathrm{A}_{1 g}$ mode has unexpectedly vanished.

22 According to LDA calculations with varying lattice 
parameters ${ }^{23}$ the relative changes of the bandwidth are approximately the same as the relative changes of the lattice parameters.

23 V. Vildosola, L. Pourovskii, R. Arita, S. Biermann, and A. Georges, Phys. Rev. B 78, 064518 (2008).

24 J. Wu and P. Phillips, arXiv:0901.3538.

25 The exact calculation of the change in harmonic zero point fluctuations gives $\delta_{r}^{2}=2.36 \cdot 10^{-5} \AA^{2}$ along the Fe-As bond and $\delta_{r}^{2}=3.81 \cdot 10^{-5} \stackrel{\circ}{2}^{2}$ along the nearest neighbor Fe-Fe bond.

26 Jinggeng Zhao, Luhong Wang, Dawei Dong, Zhiguo Liu, Haozhe Liu, Genfu Chen, Dan Wu, Jianlin Luo, Nanlin Wang, Yong Yu, Changqing Jin and Quanzhong Guo, J. Am. Chem. Soc. 130, 13828 (2008).

27 B. Lorenz, K. Sasmal, R. P. Chaudhury, X. H. Chen, R.
H. Liu, T. Wu, and C. W. Chu, Phys. Rev. B 78, 012505 (2008); M. Gooch, B. Lv, B. Lorenz, A. M. Guloy, and C.-W Chu, Phys. Rev. B 78, 180508(R) (2008); M. S. Torikachvili, S. L. Bud'ko, N. Ni, and P. C. Canfield, Phys. Rev. B 78, 104527 (2008).

28 The simplest strong coupling analysis of the SDW order on the other hand in terms of a large on-site interaction $U>t$ would have $T_{S D W} \sim J=t^{2} / U$, again a tiny but in this case negative isotope effect.

29 Parasharam M. Shirage, Kunihiro Kihou, Kiichi Miyazawa, Chul-Ho Lee, Hijiri Kito, Hiroshi Eisaki, Yasumoto Tanaka, Akira Iyo, arXiv:0903.3515 (unpublished).

${ }^{30}$ P.M. Shirage, private communication. 